\title{
THE BERGMAN KERNEL FUNCTION AND PROPER HOLOMORPHIC MAPPINGS
}

BY

\author{
STEVEN R. BELL ${ }^{1}$
}

\begin{abstract}
It is proved that a proper holomorphic mapping $f$ between bounded complete Reinhardt domains extends holomorphically past the boundary and that if, in addition, $f^{-1}(0)=\{0\}$, then $f$ is a polynomial mapping. The proof is accomplished via a transformation rule for the Bergman kernel function under proper holomorphic mappings.
\end{abstract}

1. Introduction. In one complex variable, the Bergman kernel function has long served as an essential link between boundary behavior of biholomorphic mappings and potential theory. In several complex variables, the kernel function relates biholomorphic mappings to solutions of the $\bar{\partial}$-equation. This relationship was made apparent in Fefferman's landmark paper [9], and recently in [5] and [2], the connection was found to be even more intimate.

Although the Bergman kernel has been a fruitful tool in the study of biholomorphic mappings, when the same questions were posed for proper mappings, the kernel was abandoned. Proper mappings were studied via the Kobayashi or Carathéodory metrics [10], [13], [12], [7] or by reducing the problem to one involving biholomorphic mappings [1], [8]. The purpose of this paper is to reveal that the Bergman kernel function is a potentially useful tool in the study of proper mappings, and that perhaps, in the future, questions relating to proper mappings will be answered with the same ease as those for biholomorphic mappings.

It was proved in [3] that the Bergman projections transform under proper mappings exactly as under biholomorphic mappings. In $\$ 2$ of this paper, a simplified and more natural proof of this fact will be offered. The transformation rule for the Bergman projections implies that the Bergman kernel functions also transform under proper mappings.

The transformation formula for the Bergman kernels will be exploited in the remainder of the paper. It will be shown that proper mappings between Reinhardt domains in $\mathbf{C}^{n}$ extend holomorphically past the boundaries, and that in the event that the origin is mapped to the origin with multiplicity equal to the total branching order of the mapping, the proper mapping is polynomial.

2. The transformation formula. The Bergman projection $P$ associated to a bounded domain $D$ in $\mathbf{C}^{n}$ is the orthogonal projection of $L^{2}(D)$ onto the subspace $H(D)$

Received by the editors February 3, 1981 and, in revised form, March 16, 1981.

1980 Mathematics Subject Classification. Primary 32H99; Secondary 32H10.

${ }^{1}$ Research supported by NSF grant no. MCS 80-17205.

(C)1982 American Mathematical Society 0002-9947/81/0000-1018/\$02.75 
consisting of holomorphic functions. The Bergman kernel function $K(z, w)$ is defined via

$$
P v(z)=\int_{D} K(z, w) v(w) d m_{w}
$$

for $z \in D$ and $v \in L^{2}(D)$. The kernel $K(z, w)$ is holomorphic in $z$ and antiholomorphic in $w$ and $K(w, z)=\overline{K(z, w)}$. Hence (2.1) can also be written $P v(z)=$ $\langle v, K(\cdot, z)\rangle$.

Suppose that $D_{1}$ and $D_{2}$ are two bounded domains in $\mathbf{C}^{n}$ and that $f$ is a proper holomorphic mapping of $D_{1}$ onto $D_{2}$. It is a classical fact proved by R. Remmert that $f$ is a branched cover of some finite order $m$ and that $V=\left\{f(z): \operatorname{Det}\left[f^{\prime}\right](z)=0\right\}$ is a complex variety in $D_{2}$. Let $u=\operatorname{Det}\left[f^{\prime}\right]$ and let $P_{i}$ denote the Bergman projection associated to $D_{i}$ for $i=1,2$. It is proved in [3] that the Bergman projections transform according to

$$
P_{1}(u \cdot(\phi \circ f))=u \cdot\left(\left(P_{2} \phi\right) \circ f\right)
$$

for all $\phi \in L^{2}\left(D_{2}\right)$. We now give an alternative proof of this fact.

First note that $|u|^{2}$ is equal to the real Jacobian determinant of $f$ viewed as a mapping of $\mathbf{R}^{2 n} \cong \mathbf{C}^{n}$ and hence

$$
\|u \cdot(\phi \circ f)\|_{L^{2}\left(D_{1}\right)}=m^{1 / 2}\|\phi\|_{L^{2}\left(D_{2}\right)}
$$

Furthermore, if we let $F_{1}, F_{2}, \ldots, F_{m}$ denote the $m$ inverses to $f$ which are defined locally on $D_{2}-V$, and if we write $U_{k}=\operatorname{Det}\left[F_{k}^{\prime}\right]$, then

$$
\langle v, u \cdot(\phi \circ f)\rangle_{L^{2}\left(D_{1}\right)}=\left\langle\sum_{k=1}^{m} U_{k} \cdot\left(v \circ F_{k}\right), \phi\right\rangle_{L^{2}\left(D_{2}\right)}
$$

for $v \in L^{2}\left(D_{1}\right)$ and $\phi \in L^{2}\left(D_{2}\right)$. Note that $\sum_{k=1}^{m} U_{k} \cdot\left(v \circ F_{k}\right)$ is a well-defined function in $L^{2}\left(D_{2}\right)$ even though the functions $F_{k}$ are only defined locally.

The transformation rule (2.2) is certainly true when $\phi$ is in $H\left(D_{2}\right)$. Hence, we need only check that $P_{1}(u \cdot(\phi \circ f))=0$ when $\phi \in H\left(D_{2}\right)^{\perp}$.

We now claim that the linear span of

$$
\Omega=\left\{\frac{\partial \psi}{\partial z_{i}}: \psi \in C_{0}^{\infty}\left(D_{2}-V\right) ; i=1,2, \ldots, n\right\}
$$

is a dense subspace of $H\left(D_{2}\right)^{\perp}$. Indeed, if $v \in H\left(D_{2}\right)^{\perp}$ is orthogonal to $\Omega$, then $v$ is a distributional solution to $\bar{\partial} v=0$ on $D_{2}-V$. Hence $v$ is a function in $L^{2}\left(D_{2}\right)$ which is holomorphic on $D_{2}$ minus a complex variety. At the end of this proof, we will prove an improved version of the Riemann Removable Singularity Theorem due to Bochner [6] which states that, under these circumstances, $v$ extends to be a holomorphic function on all of $D_{2}$. Therefore $v=0$. 
Hence, verifying (2.2) has been reduced to checking that $P_{1}(u \cdot(\phi \circ f))=0$ for $\phi$ in $\Omega$. But this is immediate because for $h \in H\left(D_{1}\right)$,

$$
\left\langle h, u \cdot\left(\frac{\partial \psi}{\partial z_{i}} \circ f\right)\right\rangle_{L^{2}\left(D_{1}\right)}=\sum_{k=1}^{m}\left\langle U_{k} \cdot\left(h \circ F_{k}\right), \frac{\partial \psi}{\partial z_{i}}\right\rangle_{L^{2}\left(D_{2}\right)}=0
$$

by integration by parts. The proof of the transformation rule will be completed when we have proved the

Removable Singularity Theorem. If $V$ is a complex variety in a domain $D$ and $v \in L^{2}(D)$ is holomorphic on $D-V$, then $v \in H(D)$.

Proof. For small $\varepsilon>0$, let $\chi_{\varepsilon}$ be a function in $C^{\infty}(D)$ which is supported in $V_{\varepsilon}=\{z \in D: \operatorname{dist}(z, V)<\varepsilon\}$ and such that $\chi_{\varepsilon}=1$ in a neighborhood of $V$. It is possible to construct $\chi_{\varepsilon}$ so that on a given compact subset of $D$

$$
\left|\partial \chi_{\varepsilon} / \partial z_{i}\right|<C / \varepsilon
$$

for $i=1,2, \ldots, n$ with $C$ independent of $\varepsilon$. This can be done by convolving the characteristic function for $V_{\varepsilon / 2}$ with an appropriate approximation to the identity.

We will now show that $v$ is a distributional solution to $\bar{\partial} v=0$ on $D$. Hence $v \in H(D)$. Let $\phi \in C_{0}^{\infty}(D)$. We must prove that $\left\langle v, \partial \phi / \partial z_{i}\right\rangle=0$ for $i=1,2, \ldots, n$. Let $\psi_{\varepsilon}=\partial\left(\chi_{\varepsilon} \phi\right) / \partial z_{i}$. Note that $\left\langle v, \partial \phi / \partial z_{i}\right\rangle=\left\langle v, \psi_{\varepsilon}\right\rangle$ because $v$ is holomorphic on $\operatorname{Supp}\left(1-\chi_{\varepsilon}\right)$ and $\chi_{\varepsilon}=1$ near $V$. Furthermore, the functions $\left\{\psi_{\varepsilon}\right\}_{\varepsilon>0}$ are uniformly bounded in $L^{2}(D)$ independent of $\varepsilon$. Indeed, $\left|\psi_{\varepsilon}\right|$ is $O(1 / \varepsilon)$ by $(2.3)$ and the measure of Supp $\psi_{\varepsilon}$ is $O\left(\varepsilon^{2}\right)$ because $V$ is a complex variety. Hence there is a subsequence of the functions $\left\{\psi_{\varepsilon}\right\}_{\varepsilon>0}$ with $\varepsilon \rightarrow 0$ which converge weakly to a function $\psi_{0} \in L^{2}(D)$. But clearly $\psi_{0}=0$ because Supp $\psi_{0}$ is contained in $V$ and $V$ has measure zero. Hence

$$
\left\langle v, \partial \phi / \partial z_{i}\right\rangle=\operatorname{Lim}_{\varepsilon \rightarrow 0}\left\langle v, \psi_{\varepsilon}\right\rangle=\left\langle v, \psi_{0}\right\rangle=0
$$

and the Removable Singularity Theorem is proved.

We are now in a position to prove

THeOrem 1. Suppose $D_{1}$ and $D_{2}$ are bounded domains in $\mathbf{C}^{n}$ and that $f$ is a proper holomorphic mapping of $D_{1}$ onto $D_{2}$ of order $m$. Let $u=\operatorname{Det}\left[f^{\prime}\right]$ and let $F_{1}, F_{2}, \ldots, F_{m}$ denote the $m$ local inverses to $f$ defined locally on $D_{2}-V$ where $V=\{f(z): u(z)=0\}$. Let $U_{k}=\operatorname{Det}\left[F_{k}^{\prime}\right]$ and let $K_{i}(z, w)$ denote the Bergman kernel function associated to $D_{i}$ for $i=1,2$. The Bergman kernels transform according to

$$
\sum_{k=1}^{m} K_{1}\left(z, F_{k}(w)\right) \overline{U_{k}(w)}=u(z) K_{2}(f(z), w)
$$

for all $z \in D_{1}$ and $w \in D_{2}-V$.

Of course the function on the left-hand side extends to be antiholomorphic in $w$ for all $w \in D_{2}$ by the Removable Singularity Theorem. The transformation rule was first stated in [3]. We prove it here.

Proof of Theorem 1. For $w \in D_{2}-V$, let $\theta_{w}$ be a $C^{\infty}$ function supported in $D_{2}-V$ which is radially symmetric about $w$ with $\int \theta_{w}=1$. For any function $h$ which is holomorphic on $D_{2}-V$, we have that $h(w)=\int_{D_{2}} h \overline{\theta_{w}}$. Hence $P_{2} \theta_{w}=K_{2}(\cdot, w)$. 
Now

$$
\begin{aligned}
u(z) K_{2}(f(z), w) & =u \cdot\left(\left(P_{2} \theta_{w}\right) \circ f\right)(z)=P_{1}\left(u \cdot\left(\theta_{w} \circ f\right)\right)(z) \\
& =\int_{D_{1}} K_{1}(z, \zeta) u \cdot\left(\theta_{w} \circ f\right)(\zeta) d m_{\zeta} \\
& =\sum_{k=1}^{m} \int_{D_{2}} K_{1}\left(z, F_{k}(y)\right) \overline{U_{k}(y)} \theta_{w}(y) d m_{y} \\
& =\sum_{k=1}^{m} K_{1}\left(z, F_{k}(w)\right) \overline{U_{k}(w)} .
\end{aligned}
$$

This completes the proof of Theorem 1 .

3. Proper mappings between Rienhardt domains. A domain $D$ in $\mathbf{C}^{n}$ is called a complete Reinhardt domain if whenever $z \in D$, the closed polydisc $\left\{\left|w_{j}\right| \leqslant\left|z_{j}\right|: j\right.$ $=1, \ldots, n\}$ is also contained in $D$. In such a domain $D$, the monomials $z^{\alpha}$ form a complete orthogonal system for $H(D)$ and consequently the Bergman kernel function for $D$ can be expressed

$$
K(z, w)=\sum c_{\alpha} z^{\alpha} \bar{w}^{\alpha}
$$

where $c_{\alpha}=\left\|z^{\alpha}\right\|^{-2}$. Note that $K(z, 0)=c_{0}$, a constant equal to $1 /($ volume of $D)$, and that if $|w|$ is small, then $K(z, w)$ is a holomorphic function in $z$ which extends holomorphically past the boundary of $D$ to a large neighborhood of $D$. These are two crucial facts which will be used in the proof of

THEOREM 2. If $D_{1}$ and $D_{2}$ are bounded complete Reinhardt domains, and $f$ is a proper holomorphic mapping of $D_{1}$ onto $D_{2}$, then $f$ extends to be holomorphic in $a$ neighborhood of $\overline{D_{1}}$. Furthermore, if $f(0)=0$ with multiplicity equal to the total branching order of $f$, then $f$ is a polynomial mapping.

Proof of Theorem 2. We shall use the same notation in this proof as that used in the proof of Theorem 1 . We require two key facts.

Fact 1 . If $B$ is a compact subset of $D_{1}$, then there is an open set $G$ containing $\bar{D}_{1}$ such that for $w \in B$, the function $K_{1}(z, w)$ extends to be holomorphic on $G$ as a function of $z$. If $B$ is taken to be the ball of radius $\varepsilon$ centered at the origin, then $G$ can be taken to be the ball of radius $Q / \varepsilon$ where $Q=\operatorname{Inf}\left\{|z|: z \in b D_{1}\right\}$.

Fact 2. If $f(0)=0$, then there is an integer $t$ such that $|z|^{t} \leqslant C|f(z)|$ for $z$ near the origin.

Fact 1 is a simple consequence of (3.1) and Fact 2 is a classical result on finite analytic mappings which follows from the nullstellensatz.

Let us write $K_{2}(z, w)=\sum c_{\alpha} z^{\alpha} \bar{w}^{\alpha}$. If we define $z \cdot w=\left(z_{1} w_{1}, z_{2} w_{2}, \ldots, z_{n} w_{n}\right)$ and $G(\tau)=\Sigma c_{\alpha} \tau^{\alpha}$ for $\tau \in \mathbf{C}^{n}$, then $K_{2}(z, w)=G(z \cdot \bar{w})$. Note that

$$
\frac{\partial^{\alpha}}{\partial \bar{w}^{\alpha}} K_{2}(z, w)=z^{\alpha} \frac{\partial^{\alpha} G}{\partial \tau^{\alpha}}(z \cdot \bar{w})
$$

and therefore that

$$
\frac{\partial^{\alpha}}{\partial \bar{w}^{\alpha}} K_{2}(z, 0)=\alpha ! c_{\alpha} z^{\alpha}
$$

where $c_{\alpha}$ is a nonzero constant. 
Let us write $H(z, w)=\sum_{k=1}^{m} K_{1}\left(z, F_{k}(w)\right) \overline{U_{k}(w)}$. With this notation, the transformation formula for the Bergman kernel functions becomes

$$
H(z, w)=u(z) G(f(z) \cdot \bar{w}) .
$$

If we differentiate this formula with respect to $\bar{w}$, writing $H^{\alpha}(z, w)=$ $\left(\partial^{\alpha} / \partial \bar{w}^{\alpha}\right) H(z, w)$, we obtain

$$
H^{\alpha}(z, w)=u(z) f(z)^{\alpha} \frac{\partial^{\alpha} G}{\partial \tau^{\alpha}}(f(z) \cdot \bar{w}) .
$$

If we set $w=0$ in this formula, we obtain the crucial formula

$$
H^{\alpha}(z, 0)=\alpha ! c_{\alpha} u(z) f(z)^{\alpha} .
$$

Let us now suppose that $f(0)=0$ with multiplicity equal to $m$. Then Fact 2 yields a positive integer $t$ such that $|z|^{t} \leqslant C|f(z)|$ for all $z$ in $D_{1}$. We will show that in this case, $H^{\alpha}(z, 0)$ is a polynomial, and hence, that $u \cdot f^{\alpha}$ is a polynomial for all $\alpha$. Taking $\alpha=(0, \ldots, 0)$ yields that $u$ is a polynomial, and then it follows from the unique factorization property that the components of $f$ must also be polynomials.

If $w \in D_{2}-V$ is very close to the origin, then the function $H^{\alpha}(z, w)$ extends to be a holomorphic function in $z$ defined in a large ball centered at the origin by Fact 1 . A simple normal family argument reveals that $H^{\alpha}(z, 0)$ is an entire holomorphic function of $z$. Fix $z \in \mathbf{C}^{n}$. We will produce constants $C=C(\alpha)$ and $q=q(\alpha)$ which are independent of $z$ such that $\left|H^{\alpha}(z, 0)\right| \leqslant C|z|^{q}$. This then implies that $H^{\alpha}(z, 0)$ is a polynomial. Let $r=\varepsilon|z|^{-t}$ where $\varepsilon$ is to be determined later. Let $\psi$ be a radially symmetric $C^{\infty}$ function supported in the unit ball with $\int \psi=1$. Let $\theta(w)=$ $r^{-2 n} \psi(w / r)$. Note that if $\zeta \in T=f^{-1}(\operatorname{Supp} \theta)$, then $|\zeta| \leqslant($ const $) \varepsilon^{1 / t}|z|^{-1}$. We now choose $\varepsilon$ so small that for $\zeta \in T$, the function $K_{1}(z, \zeta)$ is well defined and

$$
\operatorname{Sup}_{\zeta \in T}\left|K_{1}(z, \zeta)\right| \leqslant \text { (constant). }
$$

Note that this can be done so that the constant bound is independent of $z$. Indeed, if we set $\lambda=|z| / Q$ where $0<Q<\inf \left\{|w|: w \in b D_{1}\right\}$, then $K_{1}(z, \zeta)=K_{1}\left(\lambda^{-1} z, \lambda \zeta\right)$ by (3.1) and $|\lambda \zeta| \leqslant($ const $) \varepsilon^{1 / t} / Q$ for $\zeta \in T$. Writing $\theta_{\alpha}=\partial^{\alpha} \theta / \partial \bar{w}^{\alpha}$, we obtain

$$
\begin{aligned}
(-1)^{|\alpha|} H^{\alpha}(z, 0) & =\int_{D_{2}} H(z, w) \theta_{\alpha}(w) d m_{w} \\
& =\sum_{k=1}^{m} \int_{D_{2}} K_{1}\left(z, F_{k}(w)\right) \overline{U_{k}(w)} \theta_{\alpha}(w) d m_{w} \\
& =\int_{D_{1}} K_{1}(z, \zeta) u \cdot\left(\theta_{\alpha} \circ f\right)(\zeta) d m_{\zeta} .
\end{aligned}
$$

Hence

$$
\begin{aligned}
\left|H^{\alpha}(z, 0)\right| & \leqslant(\text { const }) \sup _{\zeta \in T}\left|K_{1}(z, \zeta)\right|\left\|u \cdot\left(\theta_{\alpha} \circ f\right)\right\|_{L^{2}\left(D_{1}\right)} \\
& \leqslant(\text { const })\left\|\theta_{\alpha}\right\|_{L^{2}\left(D_{2}\right)} \leqslant(\text { const })|z|^{t(n+|\alpha|)} .
\end{aligned}
$$

This completes the proof of the second half of the theorem. 
In the case of a general proper mapping, the formula (3.2) together with Fact 1 reveal that the functions $u \cdot f^{\alpha}$ all extend holomorphically to a common neighborhood $G$ of $\bar{D}_{1}$. This implies that $f$ extends to be holomorphic in the neighborhood $G$ because the ring of germs of holomorphic functions is a unique factorization domain. The proof of Theorem 2 is finished.

REMARKS. There are many well-known examples of proper mappings between Reinhardt domains. The proper self-mappings of the unit polydisc in $\mathbf{C}^{n}$ are precisely the mappings whose components are independent finite Blaschke products (see [14]).

If $k_{1}, k_{2}, \ldots, k_{n}$ are positive integers, the mapping which sends $\left(z_{1}, z_{2}, \ldots, z_{n}\right)$ to $\left(z_{1}^{k_{1}}, z_{2}^{k_{2}}, \ldots, z_{n}^{k_{n}}\right)$ is a proper mapping of $\left\{z: \sum_{i=1}^{n}\left|z_{i}\right|^{2 k_{i}}<1\right\}$ onto the unit ball in $\mathbf{C}^{n}$. This is an example of a proper mapping of a smooth bounded weakly pseudoconvex domain onto a smooth bounded strictly pseudoconvex domain. It is safe to conjecture that there can be no such mapping going in the opposite direction. When the domains are complete bounded Reinhardt domains, this conjecture can be verified.

THEOREM 3. Suppose that $D_{1}$ is a smooth bounded complete strictly pseudoconvex Reinhardt domain, $D_{2}$ is a smooth bounded complete Reinhardt domain, and that $f$ is a proper holomorphic mapping of $D_{1}$ onto $D_{2}$. Then $f$ is biholomorphic and extends to be a biholomorphism between larger domains $G_{1}$ and $G_{2}$ which contain $\bar{D}_{1}$ and $\bar{D}_{2}$, respectively. Hence $D_{2}$ must also be strictly pseudoconvex.

This theorem generalizes Alexander's theorem [1] which states that proper selfmappings of the unit ball are automorphisms.

Proof of Theorem 3. Let $r_{2}$ be a defining function for $D_{2}$. By Theorem $2, f$ extends smoothly to the boundary of $D_{1}$, and since the normal derivative of $f$ is nonzero by the Hopf lemma, the function $r_{1}=r_{2} \circ f$ is a defining function for $D_{1}$. We now apply an argument due to Kerzman, Kohn, and Nirenberg [11]. Since $D_{1}$ is strictly pseudoconvex, there is a large positive $\lambda$ such that $\rho=\exp \left(\lambda r_{1}\right)-1$ is a strictly plurisubharmonic defining function for $D_{1}$. Hence

$$
\operatorname{Det}\left[\frac{\partial^{2} \rho}{\partial z_{i} \partial \bar{z}_{j}}\right]=\left|\operatorname{Det}\left[f^{\prime}\right]\right|^{2}\left(\operatorname{Det}\left[\frac{\partial^{2}}{\partial z_{i} \partial \bar{z}_{j}} \exp \left(\lambda r_{2}\right)\right] \circ f\right)
$$

is greater than zero on $\bar{D}_{1}$. Therefore $\operatorname{Det}\left[f^{\prime}\right]$ cannot vanish on $\bar{D}_{1}$. Hence $f$ is an unbranched covering map. Since $D_{2}$ is simply connected, $f$ is biholomorphic. Now Theorem 2 can be applied.

It has been proved by Sunada [15] that if two Reinhardt domains are biholomorphically equivalent, then they are so via a linear mapping. Perhaps a corresponding statement is true for proper mappings: if two Reinhardt domains are related by a proper holomorphic mapping, then there is such a map which is polynomial.

Finally, we mention that the proof of Theorem 3 can be modified slightly to yield

THEOREM 4. If $D_{1}$ and $D_{2}$ are smooth bounded complete pseudoconvex Reinhardt domains contained in $\mathbf{C}^{n}$ and $f$ is a proper holomorphic mapping of $D_{1}$ onto $D_{2}$, then $f$ maps strictly pseudoconvex boundary points of $D_{1}$ to strictly pseudoconvex boundary points of $D_{2}$. 


\section{REFERENCES}

1. H. Alexander, Proper holomorphic mappings in $\mathbf{C}^{n}$, Indiana Math. J. 26 (1977), 137-146.

2. S. Bell, Biholomorphic mappings and the $\bar{\partial}$-problem, Ann. of Math. 114 (1981), 103-113.

3. Proper holomorphic mappings and the Bergman projection, Duke Math. J. 48 (1981), $167-175$.

4. S. Bell and H. Boas, Regularity of the Bergman projection in weakly pseudoconvex domains, Math. Ann. 257 (1981), 23-30.

5. S. Bell and E. Ligocka, A simplification and extension of Fefferman's theorem on biholomorphic mappings, Invent. Math. 57 (1980), 283-289.

6. S. Bochner, Weak solutions of linear partial differential equations, J. Math. Pure Appl. 35 (1956), 193-202.

7. K. Diederich and J. E. Fornaess, Proper holomorphic maps onto pseudoconvex domains with real analytic boundary, Ann. of Math. (2) 110 (1979), 575-592.

8. __ A remark on a paper by S. R. Bell, Manuscripta Math. 34 (1981), 31-44.

9. C. Fefferman, The Bergman kernel and biholomorphic mappings of pseudoconvex domains, Invent. Math. 26 (1974), 1-65.

10. G. Henkin, An analytic polyhedron is not holomorphically equivalent to a strictly pseudoconvex domain, Soviet Math. Dokl. 14 (1973), 858-862.

11. N. Kerzman, A Monge-Ampere equation in complex analysis, Proc. Sympos. Pure Math., Vol. 30, Amer. Math. Soc., Providence, R. I., 1977.

12. S. Pincuk, On proper holomorphic mappings of strictly pseudoconvex domains, Siberian Math. J. 15 (1974), 644-649.

13. R. M. Range, The Carathéodory metric and holomorphic maps on a class of weakly pseudoconvex domains, Pacific J. Math. 78 (1978), 173-189.

14. W. Rudin, Function theory in polydiscs, Benjamin, New York, 1969.

15. T. Sunada, Holomorphic equivalence problem for bounded Reinhardt domains, Math. Ann. 235 (1978), $111-128$.

Department of Mathematics, Princeton University, Princeton, New Jersey 08540 